\title{
Gamma Responses Correlate with Temporal Expectation in Monkey Primary Visual Cortex
}

\author{
Bruss Lima, ${ }^{1}$ Wolf Singer, ${ }^{1}$ and Sergio Neuenschwander ${ }^{1,2}$ \\ ${ }^{1}$ Max Planck Institute for Brain Research, D-60528 Frankfurt am Main, Germany, and ${ }^{2}$ Brain Institute, Universidade Federal do Rio Grande do Norte, \\ 59056-450 Natal, Brazil
}

Gamma oscillations have been linked to selective attention. Here, we investigate the effects of expecting a behaviorally relevant event (a change in the fixation point) on the oscillatory patterning of the local field potential and spiking responses in V1. Three protocols were used. In the first protocol, fixation point change occurred at a fixed time point, enabling predictions on task timing. In the second, fixation point change occurred in trial blocks either early or late in the trial, allowing us to compare responses during epochs of low and high expectation. Finally, we used a cue to indicate the upcoming fixation point change. All protocols led to an increase in gamma oscillations associated with alpha suppression when the monkeys expected an event in time. These effects were spatially widespread, since comparable results were observed for both central and peripheral visual representations in V1. Our findings indicate that expectations associated with perceptual decisions, motor responses, or upcoming reward may have a strong effect on the primary visual cortex, causing global, spatially nonselective modulation of gamma activity.

\section{Introduction}

It is well established that the brain uses predictive information about the world to process sensory stimuli (Grossberg, 1980; Engel et al., 2001). In a stimulus detection task, for example, performance can be considerably improved by previous experience and correct predictions. If a cue is used to indicate the likely location of a forthcoming stimulus, reaction times shorten as detection speed increases (Posner et al., 1980). Predictable stimuli are not only detected faster but also perceived more accurately. Luminance increments are better detected (Hawkins et al., 1990; Müller and Humphreys, 1991), spatial resolution increases (Balz and Hock, 1997; Yeshurun and Carrasco, 1998; Carrasco and Yeshurun, 2009), and contrast sensitivity is enhanced (Carrasco et al., 2000; Cameron et al., 2002).

Spatial cues are not, however, the only predictive information that can be used to improve performance. Information on the likely time of occurrence of expected events is equally important (Nobre et al., 2007; Coull, 2009). Temporal expectation, just as spatial attention, facilitates both stimulus detection and discrimination (Coull and Nobre, 1998; Correa et al., 2004; Nobre et al.,

Received Feb. 22, 2011; revised Aug. 28, 2011; accepted Sept. 18, 2011.

Author contributions: B.L., W.S., and S.N. designed research; B.L. and S.N. performed research; B.L. and S.N. contributed unpublished reagents/analytic tools; B.L. and S.N. analyzed data; B.L., W.S., and S.N. wrote the paper.

This work was supported by the Max Planck Society. B.L. was supported during his doctoral studies by Graduiertenkolleg, J. W. Goethe University-Frankfurt, the Max Planck Society, and the Frankfurt Institute for Advanced Studies. We thank Michaela Klinkmann and Johanna Klon-Lipok for technical assistance, and Dr. Christiane Kiefert, Yvonne Wenzel, and Clemens Sommers for animal care. We are also grateful to Pascal Fries for discussions and helpful advice on the analysis.

The authors declare no competing financial interests.

This article is freely available online through the J Neurosci Open Choice option.

Correspondence should be addressed to Sergio Neuenschwander, Brain Institute, Universidade Federal do Rio Grande do Norte, Avenida Nascimento Castro 2155, 59015-430 Natal, Brazil. E-mail: neuenschwander@neuro.ufrj.br.

DOl:10.1523/JNEUROSCI.0957-11.2011

Copyright $\odot 2011$ the authors $\quad 0270-6474 / 11 / 3115919-13 \$ 15.00 / 0$
2007). If an event that is expected to occur within a certain time window has not yet occurred, the probability of its occurrence increases with elapsing time (Nobre, 2001; Ghose and Maunsell, 2002).

Recently, Fries and collaborators have proposed a mechanism for attention based on the phase-locking of gamma oscillatory signals (Fries, 2009). The key idea is that stimulus selection is achieved by establishing stable phase relationships of spiking activity among selected populations of neurons, enabling thereby effective communication across processing levels (Fries, 2005; Womelsdorf et al., 2007). In support of his hypothesis, there is now abundant evidence showing that gamma oscillations are modulated by attention both in monkeys (Fries et al., 2001; Bichot et al., 2005; Taylor et al., 2005; Rotermund et al., 2009) and humans (Fell et al., 2002; Doesburg et al., 2008; Garcia-Garcia et al., 2010).

While the mechanisms of spatial and object attention have been intensively investigated, the neuronal basis of temporal expectation remains largely unknown. Ghose and Maunsell (2002) have shown that the attentional modulation of spiking responses in monkey V4 depends on task timing. In their study, the effects of attention on firing rate were stronger when the monkeys anticipated the behaviorally relevant stimulus. Similarly, in a visuomotor task in humans in which timing could be predicted, measurements of coherence between the motor cortex and the spinal cord have been shown to increase as function of the gosignal conditional probability (Schoffelen et al., 2005). These findings suggest that neuronal interactions performed by gamma oscillations may mediate effects of temporal expectation, although direct evidence is still lacking.

In the present study, we recorded spiking and local field potential (LFP) responses from monkey V1 during a visual detection task. Trials were designed to have a predictable time structure allowing the monkey to anticipate the fixation point 
change, prepare a motor response, and foresee the upcoming reward. Our results indicate that temporal expectations generated by predictable trial schedules strongly modulate gamma activity in V1, suggesting that coordinated activity not only is important for selection processes but also may play a role in the generation of active predictions.

\section{Materials and Methods}

Training and visual paradigm. Two male rhesus monkeys (Macaca mulatta) participated in this study. Experimental procedures were approved by local authorities (Regierungspraesidium, Hessen, Darmstadt) and were in accordance with the guidelines of the European Community (European Union Directive 86/609/EEC).

The monkeys were initially trained on a fixation task. A fixation point with $0.15^{\circ}$ diameter $\left(4 \times 4\right.$ pixels; luminance, $\left.10.0 \mathrm{~cd} / \mathrm{m}^{2}\right)$ was presented on the center of the screen. The monkeys were required to direct their gaze to the fixation point and to press a lever within $700 \mathrm{~ms}$ to initiate the trial. In a random time point between 2500 and $4000 \mathrm{~ms}$ after fixation onset, the color of the fixation point changed from red to green. To obtain a reward, the monkeys had to maintain their gaze within $1^{\circ}$ of the fixation point during the whole trial, and to release the lever within 500 $\mathrm{ms}$ after fixation point change. Trials were aborted when early or late lever releases occurred or whenever fixation was interrupted. Eye position was monitored by a search coil system (DNI; Crist Instrument; temporal resolution, $2 \mathrm{~ms}$ ), or by an infrared camera-based tracking system (developed by K. Matsuda, T. Nagami, K. Kawano, S. Yamane, Japan Science and Technology Corporation, Tsukuba, Japan; temporal resolution, $33 \mathrm{~ms}$ ). Typically, monkeys performed $\sim 1500$ correct trials in a $4 \mathrm{~h}$ session, thereby receiving their daily water requirement.

Stimuli were generated as sequences of bitmap images using an interface developed by S. Neuenschwander (LabVIEW; National Instruments) and were presented as $1024 \times 768$ pixel resolution movies running at 100 frames/s using a standard graphical board (GeForce 6600 series; NVIDIA) controlled by ActiveStim (www.activestim.com). This software allowed for high timing accuracy. The CRT monitor used for presentation (CM813ET; Hitachi) was gamma corrected to produce a linear relationship between output luminance and gray values, and subtended a visual angle of $36 \times 28^{\circ}$.

At the beginning of each recording session, a bar moving across the screen in 16 different directions was used to map the receptive fields (RFs). RF maps were obtained by computing a response matrix with 10 $\mathrm{ms}$ resolution (corresponding to $0.2^{\circ}$ in visual angle; see examples in Fig. $2 B$ ). The test stimuli consisted of moving grating or plaid stimuli. The gratings had spatial frequencies ranging from 1.25 to 2.0 cycles per degree, velocities ranging from 1.0 to $1.5 \%$, and a duty cycle of 0.3 (square gratings). These values were chosen because they elicited robust responses in V1. The plaids were constructed by superimposing two gratings with a $135^{\circ}$ moving direction offset. The gratings of higher luminance $\left(\sim 20.0 \mathrm{~cd} / \mathrm{m}^{2}\right)$ were placed on top of the one of lower luminance $\left(\sim 8.0 \mathrm{~cd} / \mathrm{m}^{2}\right)$. The visual stimulus extended from 4 to $16.0^{\circ}$ of visual angle and was positioned over the average of the receptive field centers of all recorded neurons. Stimulus location was fixed for a given protocol.

The monkeys were trained in three protocols: sequence, block, and cue protocols. In all protocols, the monkey had to release a lever upon fixation point color change. In the sequence protocol, fixation point change was fixed at $3200 \mathrm{~ms}$ relative to trial onset. The stimuli were presented in sequences (gratings-plaids-gratings or plaids-gratings-plaids). Only one of the two sequences was run during a protocol. Stimulus transitions occurred always at fixed time points (stimulus onset at $800 \mathrm{~ms}$, first stimulus change at $1600 \mathrm{~ms}$, second stimulus change at $2400 \mathrm{~ms}$ ). Since task timing was fixed, the monkeys could use stimulus transitions as a cue for predicting fixation point change. In the block protocol, fixation point change followed two schedules. It could occur early or late in the trial (at $1600 \mathrm{~ms}$ or at $3600 \mathrm{~ms}$ after trial onset, respectively). The two different schedules were presented in blocks of 50 or 160 trials. In both schedules, $15 \%$ of the trials were catch trials in which fixation point change appeared late for the early schedule, or early for the late schedule (for details, see diagram in Fig. 2A). Typically, we ran six blocks in an experimental session, whereby the two schedules alternated. For the block protocol, the stimulus remained unchanged along the trial and consisted of a single grating presented at the average preferred direction of the cells (blocks of 50 trials), or gratings presented at 16 different directions (blocks of 160 trials). In the cue protocol, we introduced a cue to indicate the upcoming fixation point change. This was achieved by a slight increase of fixation point luminance before its change in color. Cueing duration was $800 \mathrm{~ms}$. Three conditions, randomly interleaved, were used. In the first and second conditions, fixation point color change occurred early (at $1800 \mathrm{~ms}$ ) or late (at $3800 \mathrm{~ms}$ ) in the trial (cue onset at $1000 \mathrm{~ms}$ or at $3000 \mathrm{~ms}$, respectively). In the third condition (false cue), the cue appeared at the beginning of the trial (at $1000 \mathrm{~ms}$ ) while fixation point color change occurred only late (at $3800 \mathrm{~ms}$ ), at the end of the trial. Occurrence probabilities were $45 \%$ for the first and the second condition and $10 \%$ for the third condition. In this protocol, grating or plaid stimuli were used. As in the block protocol, the stimulus remained unchanged during the whole trial.

In some experiments, we manipulated the value of the reward. To this end, banana juice was used instead of water (the monkeys had a clear preference for juice), or the amount of fluid delivered was increased by a factor of 3. The two schedules were run in alternating blocks of 50 trials (typically, a total of six blocks was run in a single experiment). Task timing and visual stimulation were the same in the two schedules. Stimuli consisted of gratings, and the trial structure was the same as the one used for the early schedule in the block protocol.

Preparation and recording procedures. Each monkey was surgically implanted with a titanium bolt for fixating the head, a scleral search coil, and a titanium recording chamber. The titanium pieces were fixed to the skull by means of screws (Synthes). All surgical procedures were conducted under aseptic conditions with isoflurane anesthesia (Baxter) and assisted by a pressure-controlled ventilation unit ( $1.8 \mathrm{~L}$ per $\min \mathrm{N}_{2} \mathrm{O}$ and $0.8 \mathrm{~L}$ per min $\mathrm{O}_{2}$; Julian Station; Dräger Medical).

Recordings were made from the opercular region of V1 (receptive fields centers, $2.0-3.0^{\circ}$ eccentricity, central sites) and from the superior bank of the calcarine sulcus (10.0-13.0 eccentricity, peripheral sites). Electrodes were assembled in a customized recording device and were inserted independently into the cortex via guide tubes positioned above the dura (diameter, $300 \mu \mathrm{m}$; Ehrhardt Söhne). This device comprised five hydraulic microdrives mounted onto an $X-Y$ stage (MO-95; Narishige Scientific Instrument Laboratory), which was fixed onto the recording chamber by means of a mount adapter. Quartz-insulated tungsten-platinum electrodes (Thomas Recording; diameter, $80 \mu \mathrm{m}$ ) with impedances ranging from 0.3 to $1.0 \mathrm{M} \Omega$ were used to record simultaneously the extracellular activity from four to five sites in both superficial and deep layers of the cortex.

Data collection and spike sorting. Spiking activity of small groups of neurons [multiunit activity (MUA)] and the LFP were obtained by amplifying $(1000 \times)$ and bandpass filtering (MUA, 0.7-6.0 kHz; LFP, 0.7$170 \mathrm{~Hz}$ ) the recorded signals. For signal amplification and conditioning, we used a Plexon preamplifier connected to a HST16o25 headset (Plexon). The signals were digitized and stored using a LabVIEW-based acquisition system (SPASS; written by S. Neuenschwander). Additional $10 \times$ signal amplification was performed by the acquisition board amplifiers (E-series acquisition boards; National Instruments). Local field potentials were acquired with a resolution of $1.0 \mathrm{~ms}$. Spikes were detected by amplitude thresholding, which was set interactively after on-line visualization of the spike waveforms (typically, 2-3 SDs above noise level). Spike events and corresponding waveforms were sampled at $32 \mathrm{kS} / \mathrm{s}$ (spike waveform length, $1.2 \mathrm{~ms}$ ).

Spike sorting was performed off-line using a dynamic template matching method implemented in a custom software package [developed by Nan-Hui Chen, KunMing Institute of Zoology, KunMing, YunNan, China; see details in the study by Lima et al. (2010)].

Data analysis. For the sequence protocol, two $700 \mathrm{~ms}$ analysis windows were used. The first window started $100 \mathrm{~ms}$ after the first stimulus onset (early window), and the second window started $100 \mathrm{~ms}$ after the last stimulus onset (late window), just before fixation point change. For the block protocol, comparisons were made across $400 \mathrm{~ms}$ windows placed 
early in the trial (1200 ms after trial onset) for both the early and the late schedules. In the early schedule, the window was placed just before fixation point change, during an epoch of high expectation. In the late schedule, the window corresponded to an epoch of low expectation, as fixation point change occurred only late in the trial. For the cue protocol, the analysis window (500 ms duration) started $1300 \mathrm{~ms}$ after trial onset. For the conditions in which the cue appeared early in the trial $(1000 \mathrm{~ms}$ after trial onset, cue conditions), the window corresponded to an epoch of high expectation, since it was placed just before fixation point change. For the conditions in which the cue appeared late in the trial $(3000 \mathrm{~ms}$ after trial onset, no-cue conditions), the window corresponded to an early epoch remote from fixation point change. In this latter case, expectation was low.

Our analysis was aimed at obtaining estimates of coordinated activity in V1. Autocorrelograms for the spiking responses were computed for each trial (resolution, $1.0 \mathrm{~ms}$; time shift, $80 \mathrm{~ms}$ ) and averaged over at least 15 stimulus repetitions. A damped cosine function was fitted to the correlograms as in the study by König (1994) to obtain the modulation amplitude ratio associated with the first satellite peak in the correlogram, which estimates oscillation strength (see Fig. $1 A$ ). The peak was measured from the offset of the fitted function, and the confidence limit for the statistical significance of their values was set as follows: the $\chi^{2}$ associated with the Gabor fits had to be at least $15 \%$ smaller than the variance of the data and the $z$-scores of the peaks in the correlograms had to be $>2$.

Spectral analyses were run both for LFP and spike data using the multitaper method (Thomson, 1982) implemented in Chronux 2.0 (Mitra and Bokil, 2007), an open-source MATLAB toolbox (MathWorks; available at http://chronux.org). Essentially, the multitaper method attempts to reduce the variance of spectral estimates by premultiplying the data with several orthogonal tapers known as Slepian functions. The frequency decomposition of multitapered data segments therefore provides a set of independent spectral estimates. Mathematically, the multitapered power spectrum of a time series is defined for a given frequency as an average over all repetitions and tapers as follows:

$$
s_{x}(f)=\frac{1}{K} \sum_{k=1}^{K}\left|\tilde{x}_{n, k}(f)\right|^{2},
$$

where

$$
\tilde{x}_{n, k}(f)=\frac{1}{N} \sum_{n=1}^{N} e^{-i 2 \pi f t} w_{k}(t) x_{n}(t)
$$

is the discrete Fourier transform of the product of the measured time series sequence $\left\{x_{n}(t), n=1,2, \ldots, N\right\}$ with the $k$ th Slepian taper, denoted $w_{k}(t)$. Numerically, $\tilde{x}_{n, k}(f)$ is computed as the fast Fourier transform of the product. Data segments were padded with zeros to the length of 1024 (for the $400 \mathrm{~ms}$ and the $500 \mathrm{~ms}$ analysis window) or 2048 points (for the $700 \mathrm{~ms}$ analysis window) before the Fourier transformation. Five Slepian tapers were used for both LFP and spike data. Thus, we obtained a spectral concentration of $\pm 7.5, \pm 6.0$, and $\pm 4.3 \mathrm{~Hz}$ for the data segments of 400, 500, and $800 \mathrm{~ms}$, respectively. For computation of the spectrograms, we used windows of $200 \mathrm{~ms}$ moved at $50 \mathrm{~ms}$ steps. For this case, the spectral concentration was $\pm 15 \mathrm{~Hz}$.

Estimates of neuronal synchronization were obtained in the frequency domain by computing the coherence function, defined as follows:

$$
C_{y x}(f)=\frac{\left|S_{y x}(f)\right|}{\sqrt{S_{x}(f) S_{y}(f)}},
$$

where $S_{x}(f)$ and $S_{y}(f)$ are the multitapered power spectrum estimates of the time series $x_{n}(t)$ and $y_{n}(t)$ averaged over $n$ repetitions, respectively, and $S_{y x}(f)$ is the cross-power of these two time series. For LFP-LFP and spike-spike pairs, coherence measures were computed for data obtained from different electrodes. For LFP-spike pairs, coherence was computed for data obtained from both the same as well as from different electrodes. For a selected number of recording pairs, we performed a stratification procedure for the LFP and spike power (Schoffelen et al., 2005, 2011). The goal here was to obtain a subset of trials such that the distribution of power across trials was identical for both the low- and high-expectation conditions. To this aim, we used a routine developed by J.-M. Schoffelen (Radboud University, Nijmegen, The Netherlands) and available in the FieldTrip Toolbox (Oostenveld et al., 2011). Stratification was performed to each frequency bin individually using 10 equally spaced bins for the power distribution. Coherence for single trials was estimated using the single-trial coherence pseudovalues (STCP), as described by Womelsdorf et al. (2006), as follows:

$$
\mathrm{STCP}=N \times C_{\text {all }}-(N-1) \times C_{\text {all }-i},
$$

where $N$ is the total number of trials, $C_{\text {all }}$ is the coherence for the entire sample, and $C_{\text {all }-i}$ is the coherence for the entire sample with the $i$ th trial left out. The coherence values used to calculate the STCP were $z$-transformed, as described by Kilner et al. (2000), as follows:

$$
z=\operatorname{arctanh}(C) \times \sqrt{2 L},
$$

where the $C$ is the coherence value, and $L$ is the number of independent estimates (number of tapers $\times$ number of trials).

Baseline activity was estimated using an analysis window positioned before stimulus onset (same length as for stimulus driven activity). The LFP power was expressed in $z$-score units relative to baseline activity. For this, the power computed for the baseline activity was subtracted from the power computed for stimulus driven activity, and divided by the SD of the baseline activity. Firing rates were also computed in $z$-score units. Spiking responses were considered significant if the $z$-score value was $>1.96$ (95\% threshold). The bulk of our analyses focused on responses to the preferred stimulus eliciting maximal response. The spike power spectrum was normalized by the firing rate. The jackknife method was used to select which frequency bins between 30 and $90 \mathrm{~Hz}$ (gamma band) were significantly different when comparing the baseline with the stimulus driven activity. Gamma was computed as the average amplitude over the selected frequency bins for the power spectra and coherence functions. Delta, theta, alpha, and beta responses were equated with the average power for frequency bins between 1 and 4, 4 and 8,8 and 12, and 12 and $31 \mathrm{~Hz}$, respectively. No spectral concentration (tapering) was used to compute the power for these frequency bands.

For each site or pair of sites, we classified the selectivity for orientation and movement direction depending on the response to the grating stimuli. This classification was separately performed for the firing rate, gamma power (LFP and spike), and gamma coherence (LFPLFP, spike-spike, and LFP-spike pairs). The selectivity index was calculated as follows:

$$
\mathrm{SI}=\left(R_{\text {pref }}-R_{\text {anti }}\right) / R_{\text {pref }},
$$

where $R_{\text {pref }}$ and $R_{\text {anti }}$ represent the activity to the preferred and antipreferred stimulus configurations, respectively. To compute the selectivity index for direction, the antipreferred stimulus was defined as the one with movement direction $180^{\circ}$ away from the preferred stimulus. To determine whether there was a statistically significant relationship between neuronal activity and motion direction, we performed an ANOVA using direction of motion as the main factor. Finally, responses were fit with parametric curves based on the probability function of the von Mises distribution, which is the circular statistics analog of the normal distribution. As proposed by Swindale et al. (2003), the fitting parametric curve for direction selectivity is as follows:

$$
M(\Phi)=m+A_{1} e^{k_{1}\left(\cos \left(\Phi-\Phi_{1}\right)-1\right)}+A_{2} e^{k_{2}\left(\cos \left(\Phi-\Phi_{2}\right)-1\right)},
$$

where $\Phi$ is the grating direction of motion for which the response in being estimated, $m$ corresponds to the baseline level, $A_{1}$ and $A_{2}$ represent the maximum heights of the individual peaks, $\Phi_{1}$ and $\Phi_{2}$ are the center directions (in radians) of each peak, and $k_{1}$ and $k_{2}$, known as concentration factors, are inversely related to the width of each peak. All parameters were adjusted by a nonlinear minimization algorithm provided in the MATLAB Curve Fitting Toolbox (MathWorks). Fits that accounted for $<80 \%$ of the variance, as determined by $R^{2}$ statistics, were rejected. A 
recording site or pair of sites was classified as direction selective if all of the following criteria were fulfilled: selectivity index of $>0.5$, criterion of $p<0.05$ for the ANOVA, and $R^{2}>0.8$ for the parametric curve fit. In case the site or pair of sites was not classified as direction selective, it was tested for orientation selectivity. To this aim, activity for grating stimuli with opposite directions of motion but same orientation were pooled. The antipreferred stimulus in this case was defined as the one orthogonal to the preferred stimulus. The parametric curve fitting for orientation selectivity had $A_{2}$ constrained to equal 0 , which removed the second von Mises function from the equation. The same criteria mentioned above had to be fulfilled in order for the site or pair of sites to be classified as orientation selective.

We used the jackknife method to estimate the SEM for the power and coherence spectra (Efron and Tibshirani, 1993). For visualization purposes in the single case plots, spectral quantities were smoothed with a cubic spline function (smoothing parameter, 0.1). Group data were compared by $t$ tests (paired and independent samples) and ANOVA. Significant levels were set at $95 \%(p<0.05)$.

\section{Results}

LFPs and MUA were recorded from 363 sites in four hemispheres of two monkeys (monkey 1, 288 sites; monkey 2, 75 sites) in a total of 83 experimental sessions. For selected cases, spike sorting was applied to the MUA to obtain single-unit activity (SUA). Simultaneous recordings were made from the opercular and calcarine regions of V1 corresponding, respectively, to the central ( $\sim 3^{\circ}$ of visual angle; total of 219 sites) and the peripheral $\left(\sim 10^{\circ}\right.$ of visual angle; total of 72 sites) representations of the visual field. Correlation analyses were performed on 651 pairs of recording sites, where 427 were central-central, 62 peripheral-peripheral, and 162 central-peripheral pairs.

The monkeys were trained in three protocols: sequence, block, and cue protocols. For all protocols, the monkeys were required to hold their gaze within a window centered on a fixation point, and to respond to a change in fixation point color by a lever release (see Materials and Methods).

\section{Building expectation in stimulus sequences}

The sequence protocol consisted of three stimuli presented sequentially along the trial at fixed time points. The first and last stimuli in a sequence were always identical. The sequences could be either gratings-plaidsgratings or plaids-gratings-plaids. Because stimulus events were fixed in time and repeated precisely over hundreds of trials, the monkeys were able to predict task timing, and thus anticipate fixation point change.
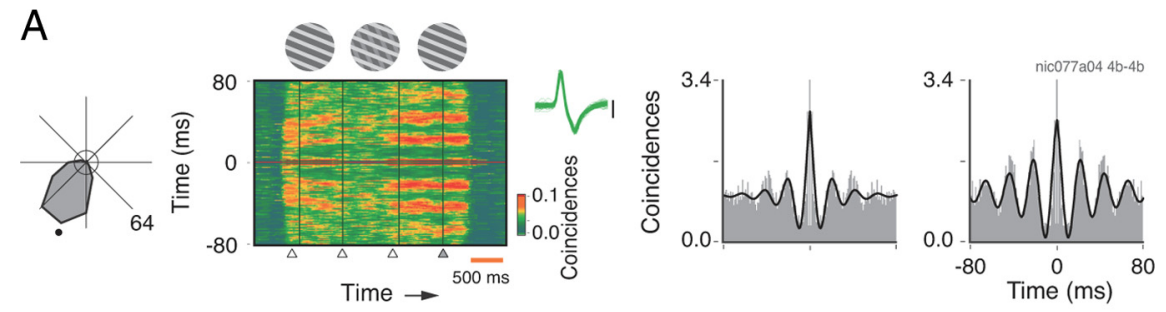

B
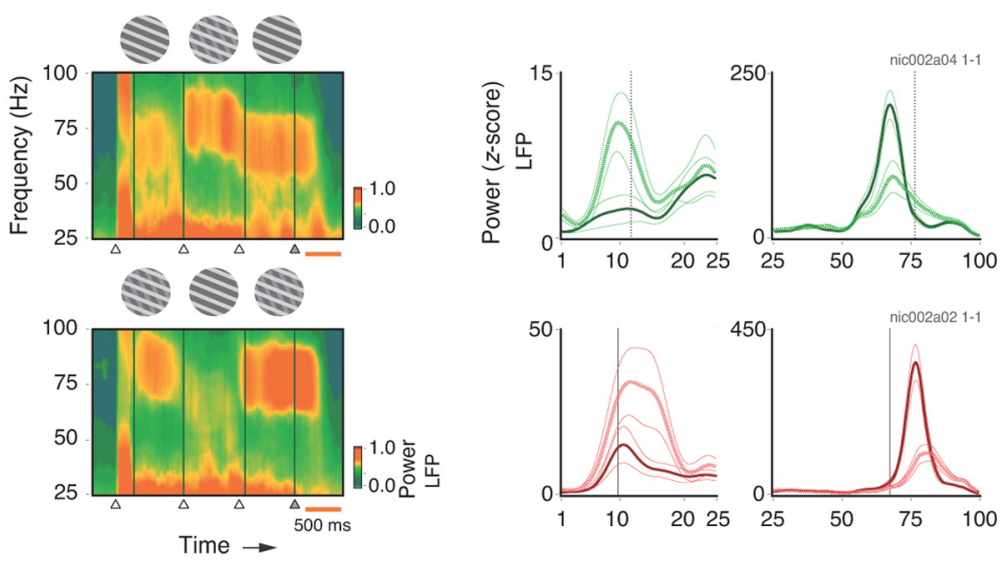

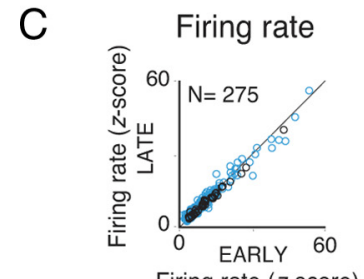

Firing rate ( $z$-score)

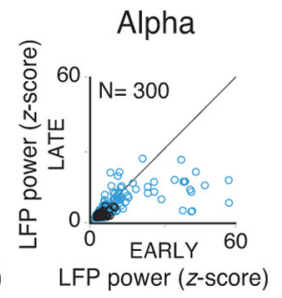

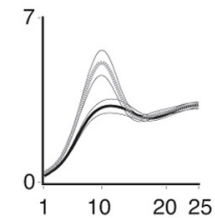

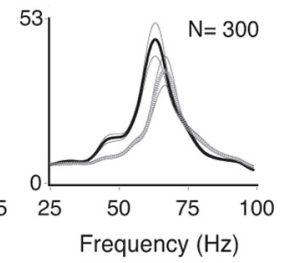

Figure 1. Spectral analysis for the sequence protocol. Comparisons were made for analysis windows placed at the early and late epochs of the trial. Stimuli were strictly the same for the two windows. A, A grating component matching the preferred direction of an isolated single unit (black dot in the tuning curve, displayed to the left) is presented throughout the trial. In the middle of the trial, however, a second grating component is presented behind the first, forming a plaid. This forms a chain of stimulus events fixed in time. Sliding window autocorrelogram analysis of a single unit recorded at $10^{\circ}$ eccentricity (spike waveforms displayed to the right) shows that gamma oscillations are more strongly induced for the late compared with the early grating in the sequence. Autocorrelograms for the early (left) and late (right) gratings show a major enhancement in gamma activity for the grating immediately preceding the change in fixation point color (modulation amplitudes of 0.75 and 1.60 , respectively). Analysis windows are indicated by boxes in $\boldsymbol{A}$. The circle in the center of the tuning curve represents the mean spontaneous rate. Task timing events (onset of early grating, onset of plaid, onset of late grating, and fixation point color change) are indicated by triangles at the bottom of the sliding window panel. A $500 \mathrm{~ms}$ calibration is shown in orange to the right. Neuronal activity before stimulus onset is due to temporal smoothing generated by the $200 \mathrm{~ms}$ sliding window used to compute the autocorrelation. Note that the visual stimulus remained on after the fixation point change event. Therefore, neuronal activity at the end of the trial persists until the monkey breaks fixation. $\boldsymbol{B}$, Time-frequency plots to the left show LFP power along the trial recorded from a single V1 opercular site for the sequences grating-plaid - grating or plaid - grating-plaid. LFP power spectra for the lower (1-25 Hz) and higher $(25-100 \mathrm{~Hz})$ frequency bands are shown to the right. Early versus late comparisons are shown in green for grating stimuliand in red for plaid stimuli (a convention used for all figures). Population power spectra (gray curves) are plotted below for comparison. The dotted and continuous lines represent the early and late phases of the trial, respectively. Opposite effects were observed for the low- and highfrequency bands. For the single case example in which early and late gratings are compared, there was a reduction of $84 \%$ in alpha power (paired $t$ test; $\mathrm{df}=8 ; p<10^{-2}$ ), but an increase of 34\% in gamma power $\left(p<10^{-2}\right)$ for the late relative to the early grating presentation. A similar result was observed for the plaid-grating-plaid sequence: a $53 \%$ decrease in alpha power (paired $t$ test; $\mathrm{df}=8$; $p=0.04)$, but a doubling in gamma power $\left(p<10^{-3}\right)$.C, Population data for firing rates ( $z$-score), LFP alpha power, LFP gamma power, and spike gamma power comparing early and late stimuli. The blue and black circles represent, respectively, sites in the central (opercular region) and peripheral (calcarine sulcus) representations of V1. Number of sites presented in each scatter plot is indicated at the top left-hand corner. A total of 300 sites was analyzed. Only those sites showing a significant increase in activity during stimulation are plotted. 
A
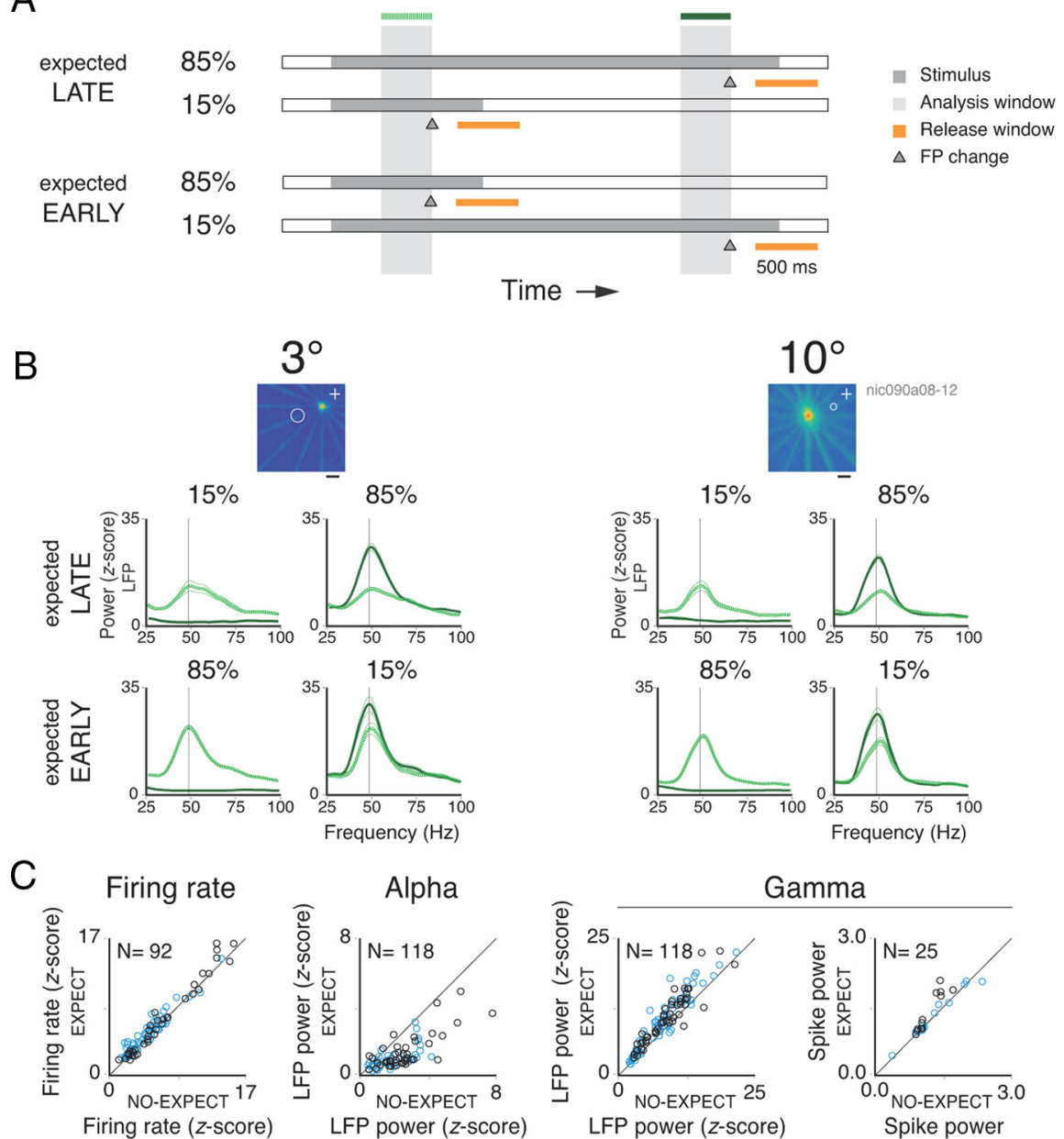

Figure 2. Spectral analysis for the block protocol. $\boldsymbol{A}$, General description of the behavioral task. $\boldsymbol{B}$, The RF maps for two sites recorded simultaneously from the central (left) and peripheral (right) representations of the visual field are shown at the top (calibration, $2^{\circ}$ ). The warmer colors, representing higher firing rates, reflect the center and extent of each RF. The fixation point position is indicated by a white cross in each map. Below each RF map are the respective LFP power spectra. The dotted and continuous lines represent $400 \mathrm{~ms}$ analysis windows positioned early or late in the trial, respectively. Note that both windows end before the fixation point change event, which takes place at $1600 \mathrm{~ms}$ for early changes and at $3600 \mathrm{~ms}$ for late changes. Both early and late windows are shown in each spectra plot for the four corresponding conditions. Comparing the spectra for all early windows, note the change in power depending on the probability of an upcoming change in the fixation point (the dotted lines on the plots above vs the dotted lines on the plots below). C, Population data for rates, LFP alpha power, LFP gamma power, and spike gamma power comparing nonexpected versus expected upcoming changes in the fixation point for the early windows. Conventions are as in Figure 1.

With this regime, behavioral performance was high (correct responses: $95 \%$ for monkey 1; $92 \%$ for monkey 2).

An example of oscillatory SUA responses recorded from peripheral V1 during a grating-plaid-grating sequence protocol is shown in Figure 1. In the sequence, the foreground grating component is presented throughout the trial, matching the preferred direction of the cell (indicated by a dot on the tuning curve plot; Fig. $1 \mathrm{~A}$ ). In the middle of the sequence, a second component appears (plaid stimuli). The single component stimulus (gratings) induced strong gamma oscillations, as shown in the sliding window autocorrelation analysis and autocorrelograms (Lima et al., 2010). Notice the strong increase in gamma for the late compared with the early stimulus, even though both were physically the same. This increase was even more evident at the LFP level.
Figure $1 B$ illustrates spectral analyses of the LFP for both gratings-plaids-gratings and plaids-gratings-plaids sequences for the same recording site (green and red curves, respectively). Consistent with the SUA data, the gamma components in the LFP show a dramatic increase for the late stimulus, regardless of stimulus sequence. This increase in gamma activity is paralleled by a decrease in alpha power. Population data are shown in Figure 1, $B$ (gray curves) and $C$. The effects of expectation are better observed when power is plotted for discrete frequency bands (Fig. 1C) than in the grand average spectra (Fig. $1 B$, bottom panels). This is due to oscillation frequency variability, which was shown to depend on various factors, such as stimulus properties, RF eccentricity, and individual (different monkeys have different spectral signatures) (Lima et al., 2010). In the plots of Figure $1 C$, data points represent $z$-score values relative to baseline (see Materials and Methods), split into central (blue) and peripheral (black) recording sites. These modulations of the LFP were highly significant for both gamma $(25 \%$ increase; paired $t$ test; $\left.\mathrm{df}=299 ; p<10^{-6}\right)$ and alpha ( $42 \%$ decrease; $\mathrm{df}=299 ; p<$ $10^{-6}$ ) oscillations. A similarly strong effect on gamma was also observed in the spike power (32\% increase; paired $t$ test; $\left.\mathrm{df}=50 ; p<10^{-6}\right)$. Despite these drastic changes in the temporal patterning of responses, there was no significant modulation in firing rates $(\mathrm{df}=274 ; p=0.28)$. Notably, there were no major differences in gamma (or alpha) modulation between central and peripheral sites (for eccentricities as large as $12^{\circ}$ ). This is interesting because the monkeys were required to direct attention to the fixation point and, thus, to the center of the visual field. Therefore, we conjecture that here the key variable was the allocation of attention in time rather than in space. The monkeys could use the regular temporal structure of the task to anticipate the fixation point change, which was the only relevant event in the task. To investigate this possibility, we used a block design protocol in which the fixation point change occurred at different but predictable time points.

\section{Building expectation in blocked trials}

In the block protocol, the fixation point change could occur early or late in the trial. These two schedules were run in alternating blocks of 50 or 160 trials. Figure $2 \mathrm{~A}$ summarizes the trial structure for the two schedules. In the late-expectation schedule, the fixation point change occurred for most of the cases (85\%) late in the trial, at $3600 \mathrm{~ms}$. Only rarely ( $15 \%$ of the cases, catch trials) did the change occur early in the trial, at $1600 \mathrm{~ms}$. Thus, the monkeys expected the fixation point change to occur in the late phase of the trial. In the early-expectation schedule, the probabil- 
ities of fixation point change were reversed (85\% early and 15\% late in the trial). These two schedules allowed us to compare neuronal responses to the same stimulus during epochs of low and high expectation.

To determine how effective our block protocol was in generating expectations, we compared reaction times for early changes in fixation point in blocks with early and late expectation. An impact of expectation on reaction times was seen for monkey 2 (mean of $403 \mathrm{~ms}$ for expected compared with $429 \mathrm{~ms}$ for nonexpected trials; paired $t$ test; $\mathrm{df}=9 ; p<10^{-3}$ ). For monkey 1 , reaction times were shorter compared with monkey 2 and showed no significant differences for expectation and nonexpectation trials (mean of $355 \mathrm{~ms} ; \mathrm{df}=11 ; p=0.9$ ). Despite these differences in performance, both monkeys showed similar increases in gamma power as a function of expectation.

Figure $2 B$ shows the LFP spectra obtained for two sites recorded simultaneously from central and peripheral V1 in monkey 1. A single moving grating stimulus was placed over the RFs to coactivate all recorded neurons. In late-expectation trials, there was a large increase in gamma power in the window placed late in the trial, compared with a window early in the trial (lateexpectation, 85\%; solid and dotted lines, respectively). Comparing responses for early windows in the trial was particularly informative, because here stimulation conditions were identical for both early- and late-expectation schedules, the only difference being the different time courses of expectation. With early expectation, gamma oscillations increased on average by 44 and $37 \%$ at 3 and $10^{\circ}$ eccentricities, respectively (two-sample $t$ test; $\mathrm{df}=453$; $p<10^{-6}$ ). Thus, similar to the sequence protocol, these effects were of comparable magnitudes at different eccentricities, suggesting a global modulation of oscillatory activity in V1. Interestingly, for the catch trials in the early-expectation schedule, when the fixation point change did not occur early but late in the trial, gamma continued to increase throughout the trial $\left(28 \%\right.$ for the $3^{\circ}$ site, paired $t$ test, $\mathrm{df}=39, p<10^{-3} ; 34 \%$ for the $10^{\circ}$ site, $\mathrm{df}=39$, $p<10^{-2}$ ), probably reflecting the growing expectation that the change was due at any time (conditional probability being maximal toward the end of the trial).

Population data for the block protocol are presented in Figure $2 C$. From a total of 118 tests for 74 recording sites ( 41 central and 33 peripheral sites, some recording sites were tested with more than one stimulus condition), we observed an average increase of $20 \%$ in LFP gamma power for windows contingent with high compared with low expectation (paired $t$ test; $\mathrm{df}=117$; $p<$ $10^{-6}$ ). This effect was equally present in the modulation of power for spiking activity (average increase of 9\%; paired $t$ test; $\mathrm{df}=24$; $p<10^{-2}$ ), although only a few sites exhibited significant oscillatory responses. Again, increase in gamma power was associated with a decrease in LFP alpha power (average decrease of 53\%; paired $t$ test; $\mathrm{df}=117 ; p<10^{-6}$ ). In contrast to the sequence protocol, however, we observed a significant increase in firing rates for the blocks associated with expectation (average increase of $11 \%$; paired $t$ test; $\left.\mathrm{df}=91 ; p<10^{-6}\right)$. No difference in the expectation effect between central and peripheral sites was, however, observed (two-sample $t$ test; $\mathrm{df}=90 ; p=0.39$ ).

Correct responses for the block protocol were $95 \%$ for monkey 1 and 96\% for monkey 2 .

\section{Cueing effects}

Temporal expectation can also be induced with cues predicting the imminent need to act. We exploited this option and increased the fixation point luminance for a duration of $800 \mathrm{~ms}$ before the occurrence of the color change (from red to green). Three con-

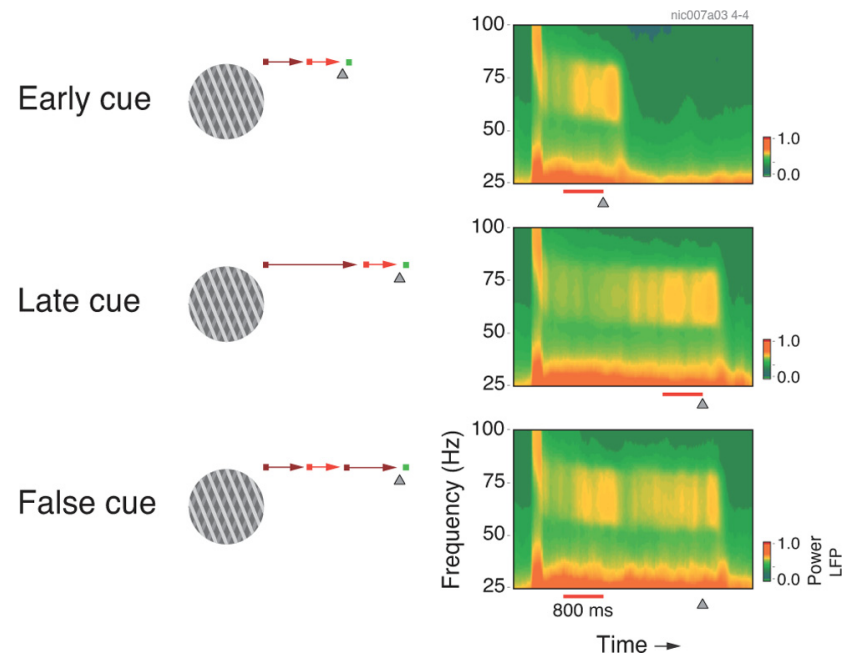

Figure 3. Time-frequency analysis of the LFP acquired during a cue protocol. Illustrated are the three conditions tested (left) with the corresponding LFP spectrogram (right). A plaid was used as visual stimulus. Enhanced LFP gamma power in the early part of the trial occurred only when the cue was presented early (early-cue and false-cue conditions). For the false-cue conditions, gamma oscillations transiently ceased when the cue was turned off, gradually increasing again toward the end of the trial.

ditions were implemented. In the first and second conditions, the cue appeared with an equal probability of $45 \%$ either early or late in the trial. In the remaining $10 \%$ of the cases (false-cue condition), the cue appeared early in the trial but the fixation point color change was delayed until the end of the trial (Fig. 3). These conditions alternated in random order and thus required cue dependent, trial-by-trial, switching of expectation to early or late phases of a trial. Correct responses for the cue protocol were $96 \%$ for monkey 1 and 92\% for monkey 2 .

The effects of cueing on gamma power are illustrated by the sliding window analysis provided in Figure 3. In this example, LFP responses were obtained to a plaid stimulus. Recordings were made from central V1 in monkey 1 . In the plots, the cueing time is indicated by a red bar (duration, $800 \mathrm{~ms}$ ). For early cueing, gamma oscillations increased shortly after cue onset. For late cueing, gamma increased only later in the trial, but this increase preceded cue onset. This is most likely due to the fact that in this case expectation increases gradually toward the end of the trial following a conditional probability function. For the false-cue condition, gamma power increased after cue presentation at the beginning of the trial, decreased after the expected color change had failed to occur, and then increased again toward the end of the trial, reflecting the increasing expectation of an imminent color change. These results are consistent with the data obtained with catch trials in the block protocol, when the fixation point change was expected to occur early but was delayed toward the end of the trial (Fig. $2 B$, catch trials).

The spectral plots in Figure $4 A$ illustrate the size of the cueing effects on LFP gamma and alpha power. Results are shown for responses to gratings (green lines) and to plaids (red lines) recorded from the same site at central V1. Analysis windows (length, $500 \mathrm{~ms}$ ) were placed at an epoch early in the trial. For the early-cue condition, the window started $300 \mathrm{~ms}$ after cue onset and ended just before the color change. The same window position was used for the late-cue condition. This allowed us to compare epochs with and without cueing for identical stimulus conditions and task timing. As described before, during conditions of high expectation, alpha was suppressed (decrease of $61 \%$ 

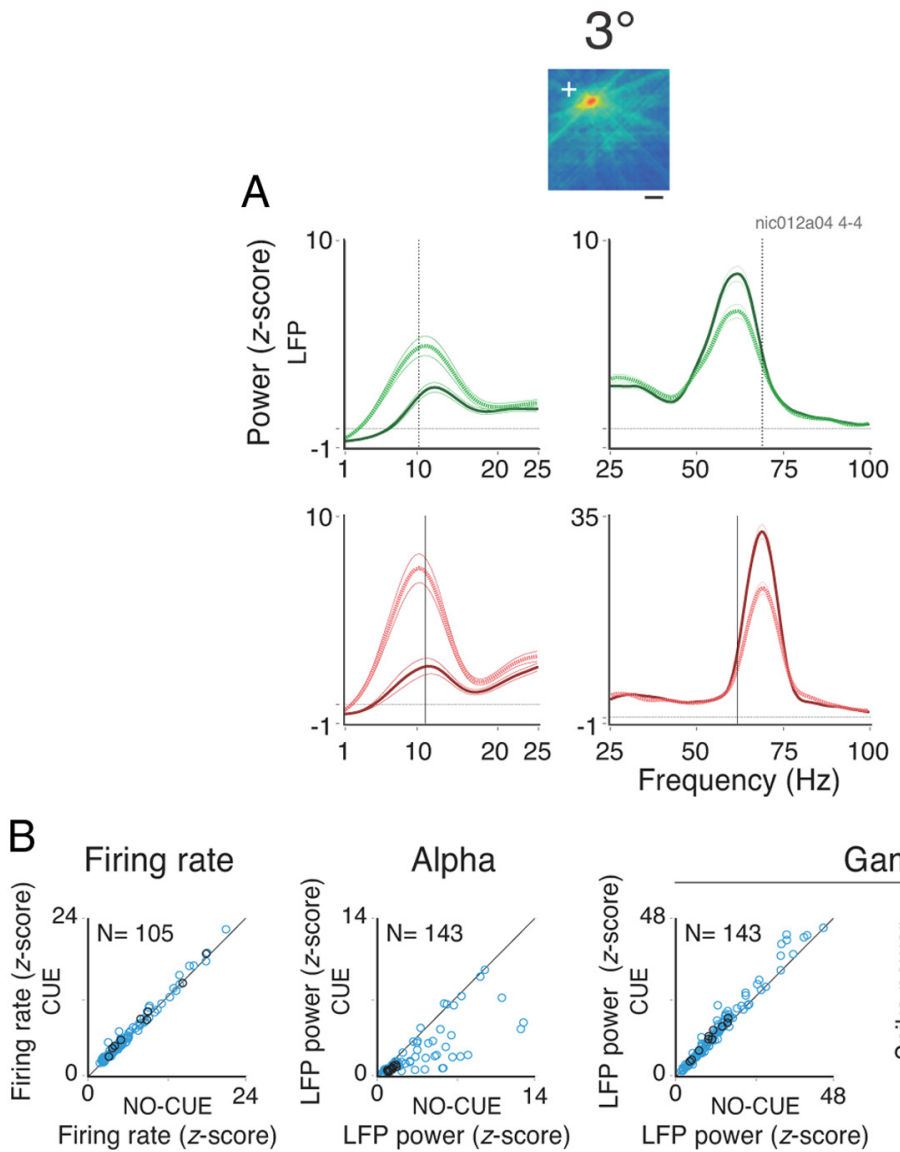

Gamma

Figure 4. Spectral analysis for the cue protocol. $A$, RF map of a site in the operculum of monkey 1 is shown at the top. LFP power spectra for the lower (1-25 Hz) and higher $(25-100 \mathrm{~Hz})$ frequency bands are shown below. The dotted and continuous lines represent the no-cue and cue conditions, respectively. As described for the two anterior protocols, opposite effects were observed for the low- and highfrequency bands. B, Population data for rates, LFP alpha power, LFP gamma power, and spike gamma power comparing no-cue with cue conditions. Data for 115 sites were acquired (11 sites recorded from the calcarine sulcus). Since some sites were tested for both the grating and plaid stimuli, a total of 144 protocols were analyzed. The blue and black circles represent, respectively, sites recorded from the central (opercular region) and peripheral (calcarine sulcus) representations of V1. The number of sites presented in each scatter plot is indicated at the top left-hand corner. Only those sites showing a significant increase in activity during stimulation are plotted. The thin lines in $\boldsymbol{A}$ correspond to the SEM.

for the gratings, two-sample $t$ test, $\mathrm{df}=359, p<10^{-6}$; decrease of $75 \%$ for the plaids, $\mathrm{df}=352, p<10^{-6}$ ) and gamma power was enhanced (increase of $15 \%$ for the gratings, two-sample $t$ test, $\mathrm{df}=359, p<10^{-2}$; increase of $23 \%$ for the plaids, $\mathrm{df}=352, p<$ $10^{-6}$ ). In accordance with our previous study (Lima et al., 2010), peak frequencies were higher for plaid compared with grating stimuli. Note that the increase in gamma power is restricted to a narrow frequency band that is centered on the peak frequency of the stimulus induced gamma oscillations. Thus, oscillation frequency was not affected.

Population data are given in Figure 4 B. On average, a 14\% increase in LFP gamma power was observed for cue compared with no-cue conditions ( paired $t$ test; $\mathrm{df}=142 ; p<10^{-6}$ ), while LFP alpha power showed an average decrease of $48 \%$ ( $\mathrm{df}=142$; $\left.p<10^{-6}\right)$. Spike gamma power increased by $2.3 \%$ for the same conditions $\left(\mathrm{df}=27 ; p<10^{-2}\right)$. Increase in firing rates was small but significant $\left(6 \%\right.$ increase; $\left.\mathrm{df}=104 ; p<10^{-6}\right)$. Possible explanations for the weak effect of expectation on spike gamma power might be due to sampling problems or to spike cycle skipping during gamma oscillations.

The modulation of power for frequencies $<30 \mathrm{~Hz}$ was similar to the one observed for the alpha band. Figure 5 shows LFP data

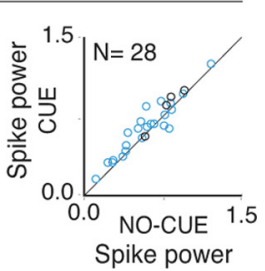

for the delta, theta, alpha, beta, and gamma bands for all three protocols (sequence, block, and cue). Observe the decrease in power for the delta, theta, and alpha bands for epochs of high expectation compared with epochs of low expectation. A similar suppression was observed for the beta band albeit of lower magnitude. Gamma activity, however, showed power enhancement for epochs contingent with high expectation.

Recently, Bosman et al. (2009) have reported that microsaccades are capable of modulating ongoing gamma oscillations in the visual cortex. We were therefore concerned that increase in gamma resulted from a different incidence of microsaccades during epochs of high expectation. For the sequence protocol, we observed a significant decrease in the frequency of microsaccades for the late (high expectation) compared with the early (low expectation) window (from an average of 1.92 to $1.60 \mathrm{microsaccades} / \mathrm{s}$; paired $t$ test, $\left.\mathrm{df}=527, p<10^{-6}\right)$. This is probably due to an improvement in fixation performance during states of high anticipation. To test whether the changes in microsaccade frequency could explain the modulation in gamma power, we selected trials having the same incidence of microsaccades for the early and late windows (195 of 528 trials). These controls were made using a high temporal and spatial resolution eye tracking system (scleral search coil system). We calculated the change in LFP gamma power for epochs of high compared with low expectation, both for the original set of trials as well as for the subset where the incidences of microsaccades were matched (same number of microsaccades for early and late windows). There was no significant difference in the modulation of LFP gamma power between the two trial sets $(\sim 18 \%$ increase in power for both sets; paired $t$ test, $\mathrm{df}=44, p=0.62$ ). Similar results were obtained for the cue protocol. Microsaccade frequency decreased for the cue compared with the no-cue condition (from an average of 1.69 to 1.35 microsaccades $/ \mathrm{s}$; two-sample $t$ test, $\left.\mathrm{df}=540, p<10^{-4}\right)$. Again, we selected a subset of trials (446 of a total of 546 trials) in which the incidence of microsaccades was matched for the cue and no-cue conditions (high and low expectation, respectively). As in the sequence protocol, there was no significant difference in LFP gamma modulation between the two trial sets ( $~ 9 \%$ increase in power for both sets; paired $t$ test, $\mathrm{df}=9, p=0.15$ ).

For all the experiments described so far, either a grating or a plaid stimulus was used to drive neuronal activity. In an additional control experiment, we investigated whether temporal expectation could still modulate neuronal activity when no stimulus was presented over the receptive fields of the recorded neurons. Figure 6 shows population data acquired using the cue protocol when no stimulus was presented. A clear modulation was only seen for the LFP alpha power, which as before was suppressed for the condition of high expectation. These findings 
suggest that it is necessary to drive neuronal activity by visual stimulation in order for the expectation effects on gamma to be revealed.

\section{Neuronal coherence}

So far, the analysis of gamma power modulation provides no direct information on neuronal interactions. Since phaselocking between oscillating neuronal groups has been suggested to be an important mechanism in neuronal communication (Fries, 2005), we also quantified synchrony among responses from pairs of recording sites. In Figure 7, we present the effects of expectation on gamma coherence. Responses to gratings recorded from two central sites in monkey 2 (electrode separation, $\sim 3 \mathrm{~mm}$ ) are shown in Figure $7 A$. Coherence is a measure which is normalized by the underlying power of the signals. It may, however, still reflect variations in oscillation strength, since epochs of high power are usually associated with smaller phase dispersions. One strategy to address this problem is to stratify for power (Schoffelen et al., 2005, 2011), a procedure that selects a subset of trials such that the distribution of power is identical across conditions. We applied this method for the power of the LFP and the power of spiking activity, which were subsequently used to compute the LFP-spike and spike-spike coherences (Fig. 7A, bottom panels). The method was used for each frequency bin $(25-100 \mathrm{~Hz})$ individually, yielding an average of 92.39 (SEM, 0.59) and 91.64 (SEM, 0.55) trials for the LFPspike and spike-spike coherence measurements, respectively. Comparisons between the low- and high-expectation conditions confirmed that their power spectra were statistically indistinguishable after stratification (channel 1, LFP, two-sample $t$ test, $\mathrm{df}=22,684, p=0.88$; channel 1 , spike, $\mathrm{df}=490, p=$ 0.94 ; channel 2, spike, $\mathrm{df}=21,362, p=0.87$ ). However, coherence for both LFP-spike and spike-spike pairs (Fig. 7A, bottom panels) still increased with expectation. Notice that changes in coherence were also not accompanied by shifts in oscillation frequency. Since we could still detect an increase in coherence after stratification, we were assured that these effects were related to neuronal interactions and not to modulations in power.

Grand-average results for all protocols are given in Figure $7 B$. Only data exhibiting significant coherence relative to baseline in the gamma band are shown (jackknife procedure of Arvesen, $p<$ $0.05)$. The average changes in coherence were highly significant (for LFP-LFP, coherence increase of $6 \%$, paired $t$ test, $\mathrm{df}=636$, $p<10^{-6}$; for LFP-spike, increase of $12 \%, \mathrm{df}=789, p<10^{-6}$; for spike-spike, increase of $15 \%, \mathrm{df}=107, p<10^{-5}$ ). Note that power stratification was not applied to all the population data shown in Figure $7 B$. The reason for this was that our results revealed robust power differences between low- and highexpectation conditions, making it difficult to find a sufficient number of pairs with matching power. For most of our experiments, we ran a relatively low number of trials. In these cases, the stratifying procedure yielded noisy coherence measurements.
Theta

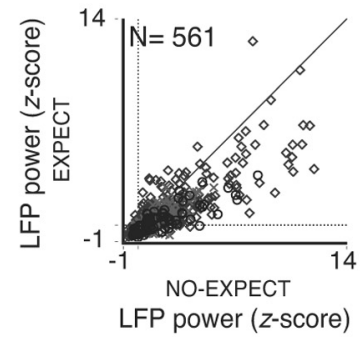

Alpha

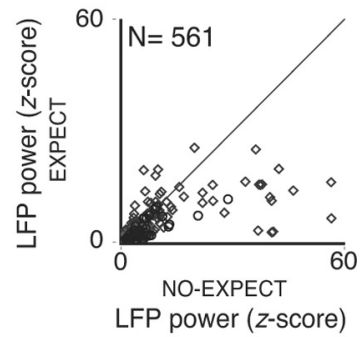

Beta

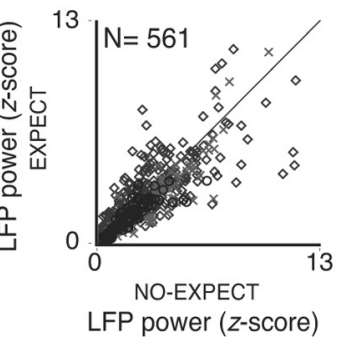

Gamma

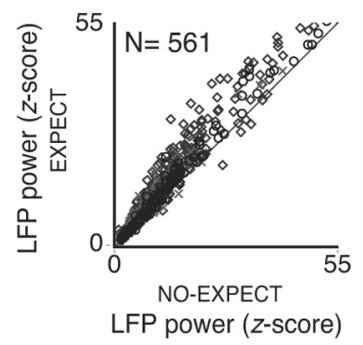

Figure 5. Population LFP power for the delta, theta, and beta frequency bands. Results for the alpha and gamma frequencies, 作 tation (average 6.3\% power decrease; paired $t$ test, $\mathrm{df}=560, p<10^{-3}$ ). The diamonds, crosses, and circles represent data points obtained for the sequence, block, and cue protocols, respectively.

Fortunately, for some experiments $(N=115$ sites $)$, the number of repetitions was sufficient for the stratification procedure (average of 170 trials for each condition). After stratification, LFP-spike and spike-spike coherence still showed a significant increase with temporal expectation (average increases of 2 and $7 \%$, respectively; two-sample $t$ test, $\mathrm{df}=202$ and 56; $p<10^{-4}$ and $p<10^{-2}$ ). LFP-LFP coherence, however, ceased to show any significant modulation by expectation after stratifying for power. This is probably because the LFP-LFP coherence modulation before stratification was already low $(2 \%)$. It is important to observe, however, that power and coherence could be mechanistically coupled in V1. In this case, epochs of high synchronization would be associated with strong oscillatory responses (Womelsdorf et al., 2007). Consequently, stratifying for power would artificially diminish any coherence modulation.

Another concern was that firing rate increases due to temporal expectation could affect the coherence modulations shown in Figure 7 (Zeitler et al., 2006). To address this point, we considered all the channels pairs included in the LFP-spike and spike-spike plots in Figure $7 B$. We selected only those pairs in which the firing rate for both electrodes decreased for epochs of high temporal expectation. The coherence modulation after controlling for firing rate increases was comparable with the one observed for the entire population shown in Figure $7 B$ (average increases of 11 and $12 \%$ for the LFP-spike and spike-spike coherences, respectively; paired $t$ test, $\mathrm{df}=346$ and $48, p<10^{-6}$ and $\left.p<10^{-2}\right)$. Thus, firing rate increases could also not account for the coherence modulations observed.

In our experiments, the recording sites were located either in the operculum (central visual representation) or in the calcarine (peripheral visual representation). This enabled us to investigate 
Firing rate

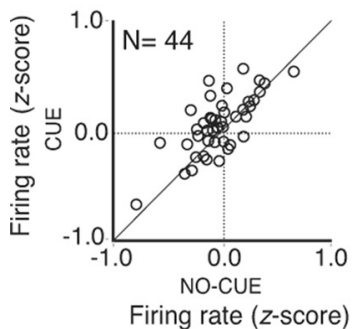

Alpha

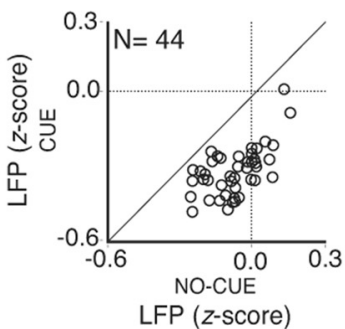

Gamma

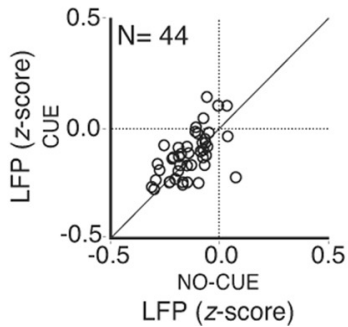

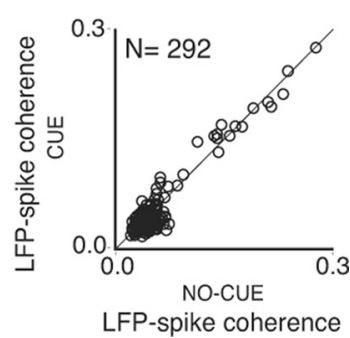

Figure 6. Effects of temporal expectation on neuronal activity when no stimulus was presented over the receptive fields of the recorded neurons. Population data comparing conditions with low (no-cue) and high (cue) expectation for the cue protocol. Analyses were performed for the firing rate, LFP alpha power, LFP gamma power, and LFP-spike coherence. For the coherence analysis, LFP-spike pairs acquired from the same as well as from different electrodes are plotted. The LFP alpha power showed a clear significant suppression for the cue compared with the no-cue condition (paired $t$ test; $\mathrm{df}=43 ; p<10^{-6}$ ). LFP gamma power (paired $t$ test; $\mathrm{df}=43 ; p=0.2$ ) and LFP-spike gamma coherence ( paired $t$ test; $\mathrm{df}=291 ; p=0.2$ ), however, showed no significant effects. Although significant differences for the firing rates could be observed (paired t test; $\mathrm{df}=43 ; p=0.001)$, no significant modulation of activity relative to baseline was found ( $z$-scores $<1.98)$. All recorded sites $(N=44)$ and LFP-spike pairs $(N=292)$ were included in the analysis. The frequency bands of $8-12$ and $30-90 \mathrm{~Hz}$ were taken as alpha and gamma activity, respectively. Data were acquired from monkeys 1 and 2.

A
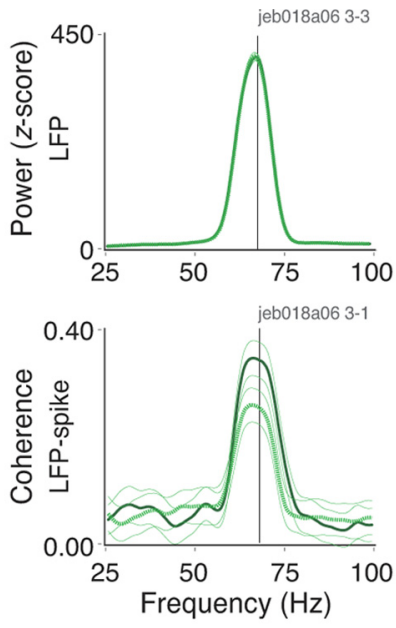

B

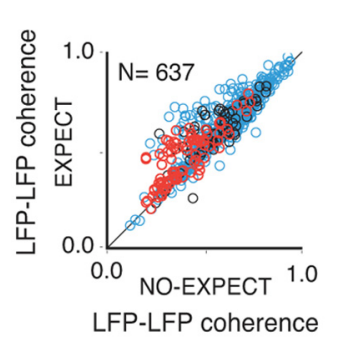

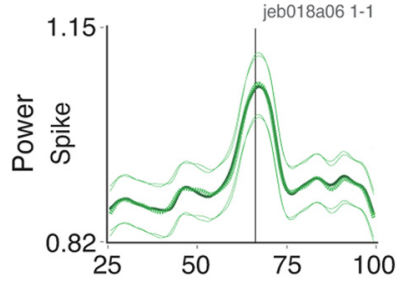

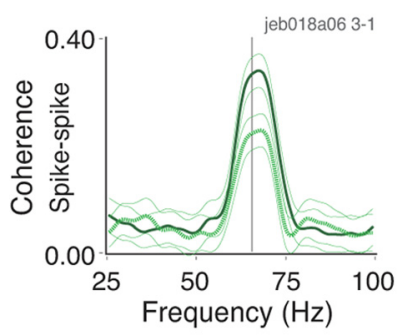

Gamma

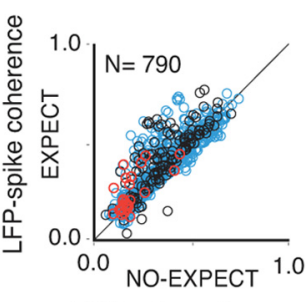

LFP-spike coherence

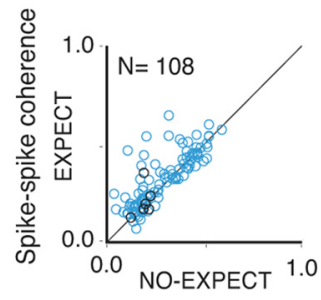

Spike-spike coherence

Figure 7. Coherence analysis of the gamma activity. A, LFP-spike and spike-spike coherence for a pair of sites recorded from the operculum of monkey 2 during a cue protocol. The spectra used to compute the coherences were stratified for power to eliminate any power modulations across the two conditions. LFP and spike gamma spectra for the corresponding sites after stratification are shown at the top. The dotted and continuous lines represent the no-cue (no-expect) and cue (expect) conditions, respectively. LFP-spike coherence exhibited an average increase of $41 \%$ (two-sample $t$ test; $\mathrm{df}=4556 ; p<10^{-6}$ ), while spike-spike coherence increased 50\% ( $\mathrm{df}=4338 ; p<$ $\left.10^{-6}\right)$, forthe expectcompared with the no-expect condition. Only those frequency bins showing a significant increase in coherence during stimulation (jackknife procedure of Arvesen, $p<0.05$ ) were selected for analysis: $61-74 \mathrm{~Hz}$ for the LFP-spike coherence and $61-73 \mathrm{~Hz}$ for the spike-spike coherence. $\boldsymbol{B}$, Population data for the sequence, block, and cue protocols. LFP-LFP, LFP-spike, and spike-spike coherence comparing no-expect versus expect conditions. The blue circles represent pairs recorded exclusively from the operculum, the black circles represent pairs recorded exclusively from the calcarine sulcus, and the red circles represent pairs that had one site located in the operculum and the other in the calcarine sulcus. Only those sites showing a significant increase in coherence during stimulation are plotted. The thin lines in $A$ correspond to the SEM.

how temporal expectation modulated neuronal interactions at different visual field eccentricities. The magnitude of coherence modulation was comparable for electrode pairs with RFs in the central and in the peripheral portions of the visual field. Central-central

and peripheral-peripheral pairs exhibited LFP-MUA coherence modulations of $10 \%$ (paired $t$ test; $\mathrm{df}=638 ; p<10^{-6}$ ) and $18 \%$ (paired $t$ test; $\mathrm{df}=122 ; p<10^{-6}$ ), respectively. The LFP-LFP coherence exhibited modulations of $5 \%$ (paired $t$ test; $\mathrm{df}=484$; $p<10^{-6}$ ) and $8 \%$ (paired $t$ test; $\mathrm{df}=63$; $p<10^{-4}$ ), respectively. Particularly pronounced were the coherence changes for pairs with different RF eccentricities. Central-peripheral pairs showed an average increase of $34 \%$ (paired $t$ test; $\mathrm{df}=27 ; p<10^{-3}$ ) in LFP-spike coherence and an increase of $17 \%$ for LFP-LFP coherence (paired $t$ test; $\left.\mathrm{df}=87 ; p<10^{-6}\right)$. These results support the notion that temporal expectation modulates neuronal interactions across a vast portion of cortex in V1.

\section{Impact on orientation selectivity}

So far, our analysis focused on responses to optimal stimuli. To investigate whether expectation also affects responses to suboptimal stimuli, we compared orientation and direction tuning plots for conditions with high and low expectation, respectively (see details in Materials and Methods). Examples of tuning curves for firing rates, LFP gamma power, and LFP-spike gamma coherence are shown in Figure $8 \mathrm{~A}$ for responses to gratings from a peripheral recording site in monkey 1 . Tuning curves based on changes in LFP gamma power showed a strong increase in orientation selectivity with expectation (increase of orientation index from 0.63 to 0.78 ). This was due to a disproportional increase of gamma power for responses to the preferred orientation. This effect was not present in the firing rates and it was moderate for the coherence. Population data confirmed these results (Fig. $8 \mathrm{~B}$ ). An average increase of $17 \%$ in selectivity was observed for the LFP gamma (paired $t$ test; $\mathrm{df}=36 ; p<$ $10^{-6}$ ). The LFP-spike coherence showed a smaller but significant $10 \%$ increase in selectivity $\left(\mathrm{df}=86 ; p<10^{-3}\right)$. Note that these changes in 
selectivity were comparable for central and peripheral sites (blue and black dots, respectively).

\section{Reward effects}

To examine whether motivational context influences the effect of expectation on V1 responses, we manipulated reward value without changing task timing. High- and low-value reward regimes were set by delivering banana juice (high value) instead of water (low value), or by changing the amount of fluid. A block design was used, with alternating blocks ( 50 trials) of highand low-reward values. Only monkey 1 was tested in this paradigm (correct responses, 97\%). Reaction times were on average $5.5 \mathrm{~ms}$ shorter for blocks of high compared with low reward value (paired $t$ test; $\mathrm{df}=12 ; p=0.04$ ), indicating that our reward schedule was effective in modulating motivation. Figure $9 A$ shows the influence of reward manipulation on the expectation effects. Responses were obtained to a grating stimulus from a central recording site. LFP alpha power was suppressed and gamma power enhanced for blocks with increased reward value. Data from a total of 45 recording sites are shown in Figure 9B. On average, alpha power decreased by $27 \%$ (paired $t$ test; $\mathrm{df}=54 ; p<10^{-6}$ ) and gamma power increased by $7 \%\left(\mathrm{df}=54 ; p<10^{-6}\right)$ in blocks with high-reward compared with those with low-reward value. Firing rates showed only a small but significant 3\% increase ( $\mathrm{df}=37 ; p=0.04)$. As before, modulations in power for central and peripheral sites were of comparable magnitude (blue and black circles, respectively).

\section{Discussion}

Our results provide the first evidence that temporal expectation modulates the power and coherence of gamma responses already at the earliest stage of cortical visual processing. It has been shown that the power and synchronization of gamma oscillations can be modulated by spatial and feature selective attention (Müller et al., 2000; Fries et al., 2001, 2008; Bichot et al., 2005; Taylor et al., 2005; Buschman and Miller, 2007). Our findings extend this notion to the temporal domain.

Fries et al. $(2001,2008)$ found that gamma synchronization in area V4 was stronger when attention was directed to a stimulus inside the RF. The expectation effects we found in V1 are of comparable magnitude as those found for spatial attention in V4. However, the effects of expectation in V1 are not confined to the attended location (here the fixation point), since the modulation in gamma was comparable for sites recorded simultaneously in the central and peripheral representations of the visual field. These findings suggest that, unlike the effects of spatial attention,
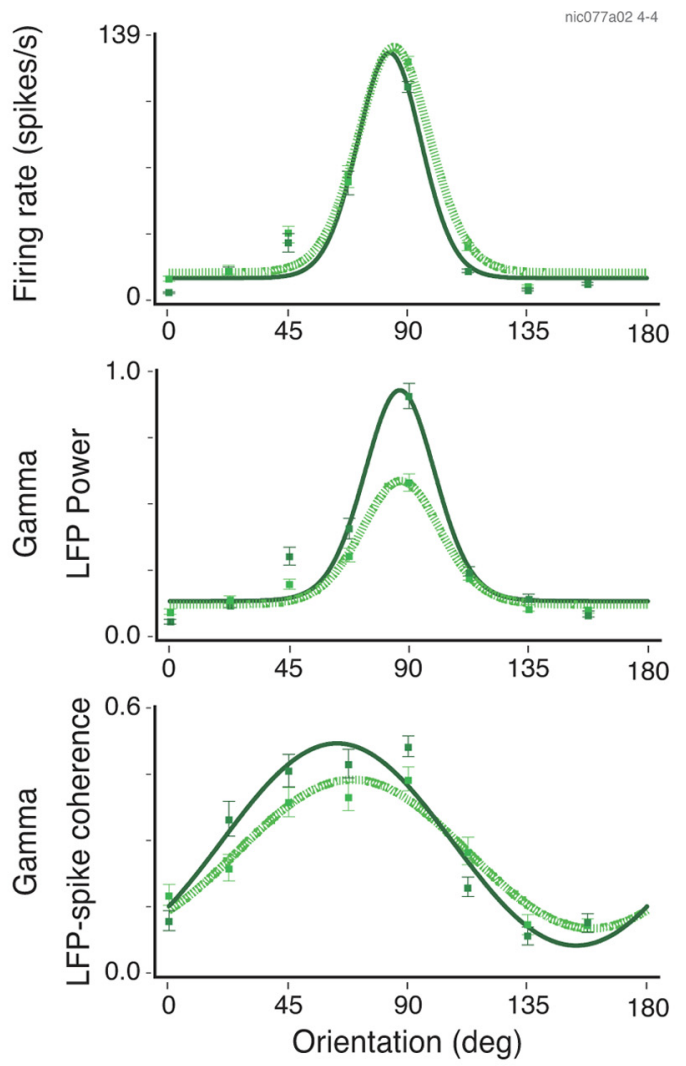

B Firing rate

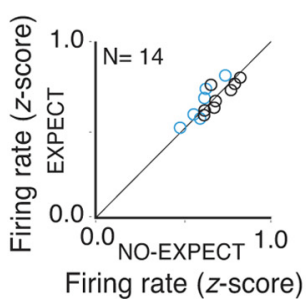

Figure 8. Changes in selectivity with temporal expectation. $\boldsymbol{A}$, The firing rate, LFP gamma power, and LFP-spike gamma coherence for a site in calcarine sulcus of monkey 1 during a grating-plaid-grating sequence protocol. Neuronal activity for the various stimulus configurations could be fitted with a single von Mises function in all three analyses and was therefore classified as orientation selective ( $R^{2}>0.9$ for all cases). The dotted and continuous lines indicate activity for the early (low expectation) and late (high expectation) analysis windows, respectively. The firing rate responses ( $\boldsymbol{A}$, top panel) for the eight orientations tested were very similar during both periods (orientation index of $\sim 0.9$ computed from the fitted von Mises function). For the LFP power, however, enhancement in gamma activity during the high-expectation period took place predominantly for the preferred orientation ( $\sim 90^{\circ} ; \boldsymbol{A}$, middle panel). Enhanced gamma phase-locking also occurred mainly for the preferred orientation $\left(\sim 70^{\circ} ; \boldsymbol{A}\right.$ bottom panel). $\boldsymbol{B}$, Population data comparing the selectivity indexes during periods of low and high expectation. Analysis for the firing rate, LFP gamma power and LFP-spike gamma coherence are shown. Only cases in which activity significantly increased with expectation are plotted ( $t$ test for the comparison between low- and high-expectation periods). The blue and black circles represent sites or pairs of sites recorded from the operculum and calcarine sulcus, respectively. The red circles represent pairs in which one electrode was placed in the operculum and the other in the calcarine sulcus. The error bars in $\boldsymbol{A}$ correspond to the SEM. the effects of temporal expectation are global. Modulation in firing rates due to attention are known to be weak in early visual areas, increasing in strength over hierarchical levels (Mehta et al., 2000; Maunsell and Cook, 2002). Similarly, subdural LFP recordings in humans have shown that the effects of spatial attention in V1 on the event-related potential are rather small (Yoshor et al., 2007). The comparatively strong effects of temporal expectation and their global nature indicate that the underlying mechanisms differ from those of spatial attention. Further experiments are needed to assess the relative contribution of attention in space and time, respectively, on neuronal responses in V1. 
A
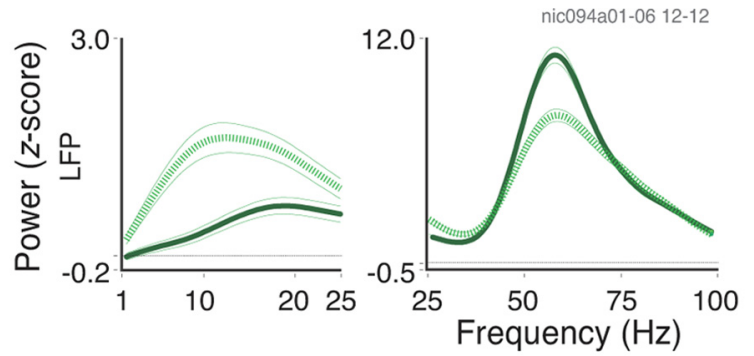
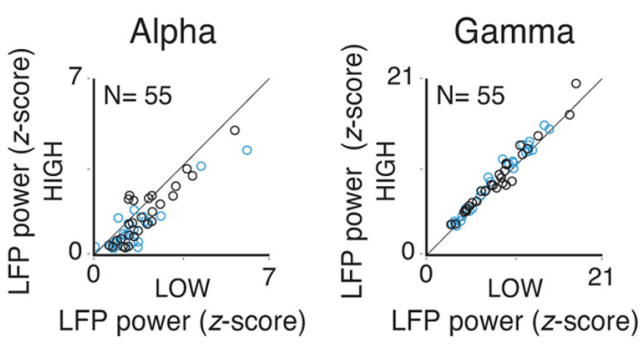

Figure 9. Effect of reward value expectation. $A$, LFP power recorded from a site in the operculum. Low-frequency $(1-25 \mathrm{~Hz})$ and high-frequency $(25-100 \mathrm{~Hz}$ ) band spectra responded in opposite ways to the expectation of reward value. The dotted and continuous lines represent conditions in which low- and high-reward value were expected, respectively. Alpha activity decreased on average $86 \%$ (two-sample $t$ test; $\mathrm{df}=440 ; p<10^{-6}$ ), while gamma activity was enhanced by $17 \%$ (two-sample $t$ test; $\mathrm{df}=$ $440 ; p<10^{-4}$ ). B, Population data for rates, LFP alpha power, and LFP gamma power comparing low-value and high-value conditions. The blue and black circles represent sites recorded from the central (opercular region) and peripheral (calcarine sulcus) representations of $\mathrm{V} 1$, respectively. For some sessions, a second grating stimulus with different direction of movement was run for the same set of recording sites. This gave rise to a total of 55 data points used in the population analysis. Only those sites showing a significant increase in activity during stimulation are plotted. Number of data points presented in each scatter plot is indicated at the top left-hand corner. The thin lines in $\boldsymbol{A}$ correspond to the SEM.

The effects of temporal expectation were not restricted to the gamma band. The increase in gamma power was systematically associated with alpha suppression. Fries et al. (2008) also reported that attention suppresses alpha activity in V4. Several other reports in monkeys and humans have shown that alpha suppression is involved in a variety of cognitive processes, including attention and memory (for review, see Klimesch et al., 2007), suggesting that a trade-off between alpha and gamma activity may control information flow in the brain (Jokisch and Jensen, 2007).

A central finding in our study is that gamma oscillation strength changes along the trial as a function of the conditional probability of occurrence of the behaviorally relevant event (hazard rate). In a time-frequency analysis of data from Fries et al. (2001), Liang et al. (2005) obtained results similar to ours, suggesting that hazard rates may correlate with modulation of gamma power. In contrast to their study, which was limited to only one time schedule, we used different protocols to allow the monkey to generate probabilistic time schedules of fixation point change. Here, information about task timing could be obtained either from a cue or from the consistency in the time schedule of the blocked trials. All paradigms led essentially to the same results, namely an increase in gamma associated with alpha suppression when the monkeys were attending to an event with predictable timing. Similar findings were obtained in the study by Schoffelen et al. (2005) for corticospinal synchronization in humans, showing that coherence for the gamma band closely followed the hazard rate of stimulus change probability. The authors' interpretation was that gamma synchronization boosts neuronal communication during states demanding allocation of resources because they found that reaction times shortened for trials in which coherence was high. Comparable results were obtained in V4 for a spatial attention task (Womelsdorf et al., 2006). We have also observed a significant shortening of reaction times, albeit in only one monkey, for conditions of high expectation.

Ghose and Maunsell (2002) have shown that attentional effects may vary depending on the hazard rate of stimulus change. Thus, anticipation can modulate neuronal activity, in parallel to spatial attentional effects. Our study extends these findings by providing evidence that expectation effects exhibit fast dynamics. In the study by Ghose and Maunsell (2002), the monkeys were trained extensively on each task separately, making it impossible to compare the responses from the same cell for the two conditions. In our block protocol, by contrast, the two time schedules were alternated several times during the same recording, using blocks as short as 30 trials, indicating that the monkeys could rapidly adjust their expectation to a new context.

In the present study, the anticipatory effects in gamma power and coherence were apparent only in the presence of visual input. When the cue protocol was run without a visual stimulus (Fig. 6), we detected no significant modulation of gamma activity, although an alpha suppression was still visible. This suggests that expectation induced a covert state change that became manifest only once cortical circuits were activated by the stimulus. A few studies have shown anticipatory effects on neuronal synchronization in the absence of visual stimulation. de Oliveira et al. (1997) have shown an increase in neuronal synchronization in MT for the period before stimulus onset. The authors attributed this effect to expectation, although this interpretation is arguable since the prestimulus period was variable. Roelfsema et al. (1997) found in the behaving cat that LFP correlations between cortical areas in the beta band occurred already in the trial epoch preceding presentation of the relevant stimulus after the animals had been alerted by a sound cue indicating the onset of the trial. A recent study in V1 (Sirotin and Das, 2009) has shown an anticipatory increase of the intrinsic hemodynamic signal responses while the monkeys engaged in a periodic task. Interestingly, simultaneous recording of neuronal activity revealed no modulation of LFP gamma power or firing rates. This finding suggests that covert state changes in the cortex associated with expectation may be visible in the hemodynamical signal, but not in the LFP or spiking activity. Together, these observations point to the importance of temporal expectation in early sensory processing. Thus, when studying awake subjects it will be important to distinguish between effects of focused attention and temporal expectation.

It remains to be explained how temporal expectation may influence oscillatory activity over large extensions of the visual cortex at relatively short timescale $(<1 \mathrm{~s})$. A possible explanation could be fluctuations of central states such as arousal. In accord with this hypothesis, it has been shown that the gamma oscillations increase with heightened states of central activation (Herculano-Houzel et al., 1999). Of the numerous modulatory systems that control central states, cholinergic projections have been shown to enhance gamma oscillations. Acetylcholine facilitates gamma oscillations in vitro (Buhl et al., 1998) and in vivo 
(Rodriguez et al., 2004). Cholinergic projections from the basal forebrain have widely distributed terminal fields and activate the cortex in a rather global manner (Sarter and Bruno, 2000; Jones, 2008). Thus, they could mediate the widespread effects of anticipation. A contribution of other modulatory systems should not be excluded, however, since they exhibit a similarly global organization and can change their activity with changes in central states. The fact that the expectation-dependent increase in gamma power was modulated in addition by reward value does indeed suggest that modulatory influences other than arousal were involved.

We propose that the anticipatory enhancement of gamma activity in V1 serves to prepare the cortical networks to respond. This preparation is likely to involve task-specific dynamical coordination of large-scale networks comprising both sensory and executive areas (Roelfsema et al., 1997). Coherent gamma oscillations could represent an efficient mechanism to insure communication between different regions in the brain because (1) they are associated with enhanced synchronization of spikes, which facilitates propagation of activity across sparsely connected networks (Bruno and Sakmann, 2006); and (2) phase adjustments of oscillatory activity allow for selective routing of responses in distributed recurrent networks such as the cortex (Moser et al., 2010). Future studies will have to clarify whether the effects described here are a consequence of general arousal, affecting equally all stages, or whether they are a reflection of task-specific dynamic coordination. To distinguish between these possibilities, simultaneous recordings would have to be performed from multiple cortical areas while subjects prepare to respond in a task allowing for predictions in time.

\section{References}

Balz GW, Hock HS (1997) The effect of attentional spread on spatial resolution. Vision Res 37:1499-1510.

Bichot NP, Rossi AF, Desimone R (2005) Parallel and serial neural mechanisms for visual search in macaque area V4. Science 308:529-534.

Bosman CA, Womelsdorf T, Desimone R, Fries P (2009) A microsaccadic rhythm modulates gamma-band synchronization and behavior. J Neurosci 29:9471-9480.

Bruno RM, Sakmann B (2006) Cortex is driven by weak but synchronously active thalamocortical synapses. Science 312:1622-1627.

Buhl EH, Tamás G, Fisahn A (1998) Cholinergic activation and tonic excitation induce persistent gamma oscillations in mouse somatosensory cortex in vitro. J Physiol 513:117-126.

Buschman TJ, Miller EK (2007) Top-down versus bottom-up control of attention in the prefrontal and posterior parietal cortices. Science 315:1860-1862.

Cameron EL, Tai JC, Carrasco M (2002) Covert attention affects the psychometric function of contrast sensitivity. Vision Res 42:949-967.

Carrasco M, Yeshurun Y (2009) Covert attention effects on spatial resolution. Prog Brain Res 176:65-86.

Carrasco M, Penpeci-Talgar C, Eckstein M (2000) Spatial covert attention increases contrast sensitivity across the CSF: support for signal enhancement. Vision Res 40:1203-1215.

Correa A, Lupiáñez J, Milliken B, Tudela P (2004) Endogenous temporal orienting of attention in detection and discrimination tasks. Percept Psychophys 66:264-278.

Coull JT (2009) Neural substrates of mounting temporal expectation. PLoS Biol 7:e1000166.

Coull JT, Nobre AC (1998) Where and when to pay attention: the neural systems for directing attention to spatial locations and to time intervals as revealed by both PET and fMRI. J Neurosci 18:7426-7435.

de Oliveira SC, Thiele A, Hoffmann KP (1997) Synchronization of neuronal activity during stimulus expectation in a direction discrimination task. J Neurosci 17:9248-9260.

Doesburg SM, Roggeveen AB, Kitajo K, Ward LM (2008) Large-scale gamma-band phase synchronization and selective attention. Cereb Cortex 18:386-396.
Efron B, Tibshirani R (1993) An introduction to the bootstrap. New York: Chapman and Hall.

Engel AK, Fries P, Singer W (2001) Dynamic predictions: oscillations and synchrony in top-down processing. Nat Rev Neurosci 2:704-716.

Fell J, Klaver P, Elger CE, Fernández G (2002) Suppression of EEG gamma activity may cause the attentional blink. Conscious Cogn 11:114-122.

Fries P (2005) A mechanism for cognitive dynamics: neuronal communication through neuronal coherence. Trends Cogn Sci 9:474-480.

Fries P (2009) Neuronal gamma-band synchronization as a fundamental process in cortical computation. Annu Rev Neurosci 32:209-224.

Fries P, Reynolds JH, Rorie AE, Desimone R (2001) Modulation of oscillatory neuronal synchronization by selective visual attention. Science 291:1560-1563.

Fries P, Womelsdorf T, Oostenveld R, Desimone R (2008) The effects of visual stimulation and selective visual attention on rhythmic neuronal synchronization in macaque area V4. J Neurosci 28:4823-4835.

Garcia-Garcia M, Yordanova J, Kolev V, Domínguez-Borràs J, Escera C (2010) Tuning the brain for novelty detection under emotional threat: the role of increasing gamma phase-synchronization. Neuroimage 49:1038-1044.

Ghose GM, Maunsell JH (2002) Attentional modulation in visual cortex depends on task timing. Nature 419:616-620.

Grossberg S (1980) How does the brain build a cognitive code? Psychol Rev $87: 1-51$.

Hawkins HL, Hillyard SA, Luck SJ, Mouloua M, Downing CJ, Woodward DP (1990) Visual attention modulates signal detectability. J Exp Psychol Hum Percept Perform 16:802-811.

Herculano-Houzel S, Munk MH, Neuenschwander S, Singer W (1999) Precisely synchronized oscillatory firing patterns require electroencephalographic activation. J Neurosci 19:3992-4010.

Jokisch D, Jensen O (2007) Modulation of gamma and alpha activity during a working memory task engaging the dorsal or ventral stream. J Neurosci 27:3244-3251.

Jones BE (2008) Modulation of cortical activation and behavioral arousal by cholinergic and orexinergic systems. Ann N Y Acad Sci 1129:26-34.

Kilner JM, Baker SN, Salenius S, Hari R, Lemon RN (2000) Human cortical muscle coherence is directly related to specific motor parameters. J Neurosci 20:8838-8845.

Klimesch W, Sauseng P, Hanslmayr S (2007) EEG alpha oscillations: the inhibition-timing hypothesis. Brain Res Rev 53:63-88.

König P (1994) A method for the quantification of synchrony and oscillatory properties of neuronal activity. J Neurosci Methods 54:31-37.

Liang H, Bressler SL, Buffalo EA, Desimone R, Fries P (2005) Empirical mode decomposition of field potentials from macaque $\mathrm{V} 4$ in visual spatial attention. Biol Cybern 92:380-392.

Lima B, Singer W, Chen NH, Neuenschwander S (2010) Synchronization dynamics in response to plaid stimuli in monkey V1. Cereb Cortex 20:1556-1573.

Maunsell JH, Cook EP (2002) The role of attention in visual processing. Philos Trans R Soc Lond B Biol Sci 357:1063-1072.

Mehta AD, Ulbert I, Schroeder CE (2000) Intermodal selective attention in monkeys. I: Distribution and timing of effects across visual areas. Cereb Cortex 10:343-358.

Mitra P, Bokil H (2007) Observed brain dynamics. Oxford, UK: Oxford UP. Moser EI, Corbetta M, Desimone R, Frégnac Y, Fries P, Graybiel AM, Haynes JD, Itti L, Melloni L, Monyer H, Singer W, von der Malsburg C, Wilson MA (2010) Coordination in brain systems. In: Dynamical coordination in the brain. From neurons to mind (von der Malsburg C, Phillips WA, Singer W, eds), pp 193-214. Cambridge, MA: MIT.

Müller HJ, Humphreys GW (1991) Luminance-increment detection: capacity-limited or not? J Exp Psychol Hum Percept Perform 17:107-124.

Müller MM, Gruber T, Keil A (2000) Modulation of induced gamma band activity in the human EEG by attention and visual information processing. Int J Psychophysiol 38:283-299.

Nobre AC (2001) Orienting attention to instants in time. Neuropsychologia 39:1317-1328.

Nobre A, Correa A, Coull J (2007) The hazards of time. Curr Opin Neurobiol 17:465-470.

Oostenveld R, Fries P, Maris E, Schoffelen JM (2011) FieldTrip: open source software for advanced analysis of MEG, EEG, and invasive electrophysiological data. Comput Intell Neurosci 2011:156869. 
Posner MI, Snyder CR, Davidson BJ (1980) Attention and the detection of signals. J Exp Psychol 109:160-174.

Rodriguez R, Kallenbach U, Singer W, Munk MH (2004) Short- and longterm effects of cholinergic modulation on gamma oscillations and response synchronization in the visual cortex. J Neurosci 24:10369-10378.

Roelfsema PR, Engel AK, König P, Singer W (1997) Visuomotor integration is associated with zero time-lag synchronization among cortical areas. Nature 385:157-161.

Rotermund D, Taylor K, Ernst UA, Kreiter AK, Pawelzik KR (2009) Attention improves object representation in visual cortical field potentials. J Neurosci 29:10120-10130.

Sarter M, Bruno JP (2000) Cortical cholinergic inputs mediating arousal, attentional processing and dreaming: differential afferent regulation of the basal forebrain by telencephalic and brainstem afferents. Neuroscience 95:933-952.

Schoffelen JM, Oostenveld R, Fries P (2005) Neuronal coherence as a mechanism of effective corticospinal interaction. Science 308:111-113.

Schoffelen JM, Poort J, Oostenveld R, Fries P (2011) Selective movement preparation is subserve by selective increases in corticomuscular gammaband coherence. J Neurosci 31:6750-6758.

Sirotin YB, Das A (2009) Anticipatory haemodynamic signals in sensory cortex not predicted by local neuronal activity. Nature 457:475-479.
Swindale NV, Grinvald A, Shmuel A (2003) The spatial pattern of response magnitude and selectivity for orientation and direction in cat visual cortex. Cereb Cortex 13:225-238.

Taylor K, Mandon S, Freiwald WA, Kreiter AK (2005) Coherent oscillatory activity in monkey area V4 predicts successful allocation of attention. Cereb Cortex 15:1424-1437.

Thomson D (1982) Spectrum estimation and harmonic analysis. Proc IEEE 70:1055-1096.

Womelsdorf T, Fries P, Mitra PP, Desimone R (2006) Gamma-band synchronization in visual cortex predicts speed of change detection. Nature 439:733-736.

Womelsdorf T, Schoffelen JM, Oostenveld R, Singer W, Desimone R, Engel AK, Fries P (2007) Modulation of neuronal interactions through neuronal synchronization. Science 316:1609-1612.

Yeshurun Y, Carrasco M (1998) Attention improves or impairs visual performance by enhancing spatial resolution. Nature 396:72-75.

Yoshor D, Ghose GM, Bosking WH, Sun P, Maunsell JH (2007) Spatial attention does not strongly modulate neuronal responses in early human visual cortex. J Neurosci 27:13205-13209.

Zeitler M, Fries P, Gielen S (2006) Assessing neuronal coherence with single-unit, multi-unit, and local field potential. Neural Comput 18:2256-2281. 